\title{
Cellular apoptosis susceptibility protein and proliferation in human hepatocellular carcinoma
}

\author{
KATSUYA SHIRAKI, KATSUHIKO FUJIKAWA, KAZUSHI SUGIMOTO, \\ TAKESHI ITO, TAKENARI YAMANAKA, MASAHIRO SUZUKI, KENTARO YONEDA, \\ KAZUSHI SUGIMOTO, KOUJIRO TAKASE and TAKESHI NAKANO
}

First Department of Internal Medicine, Mie University School of Medicine, Tsu, Mie 514-8507, Japan

Received January 27, 2006; Accepted March 15, 2006

\begin{abstract}
The cellular apoptosis susceptibility protein (CAS) is the human homologue of the product of the essential yeast chromosome segregation gene, CSE1, and has important roles in tumor necrosis factor (TNF)-induced apoptosis and cell proliferation. In this study, we used immunoblotting and immunohistochemistry to look at CAS expression in human hepatocellular carcinoma (HCC) cells. We also sudied the correlation between CAS expression and cell proliferation. To do this, we studied the expression of proliferating cell nuclear antigen (PCNA) by immunostaining and at apoptosis by in situ nick end-labeling (TUNEL), followed by calculation of the PCNA labeling index (PCNA LI) and TUNEL labeling index (TUNEL LI). CAS was constitutively expressed in human HCC cell lines and was primarily confined to the cytoplasm of the cells. PCNA LI and TUNEL LI were significantly higher in HCC than in non-tumor tissue $(\mathrm{p}<0.01)$; however, the ratio of TUNEL LI/PCNA LI in HCC was significantly lower than that of non-tumor tissue. Immunohistochemistry revealed that the staining intensity score of CAS in HCC was significantly higher than that of non-tumor tissue $(p<0.05)$. These results indicate that there is an augmentation of pro-liferative activity and apoptosis in HCC tissue, as compared to non-tumor tissue. There was a significant positive correlation between CAS and PCNA LI $(\mathrm{p}<0.05)$. In addition, we observed an inverse relationship between CAS expression and TUNEL LI, although the correlation did not reach statistical significance. These results suggest that CAS is expressed at higher levels in human HCC tissue than in non-tumor tissue. CAS may be associated with cell proliferation rather than apoptosis, and further, CAS
\end{abstract}

Correspondence to: Dr Katsuya Shiraki, First Department of Internal Medicine, Mie University School of Medicine, 2-174 Edobashi, Tsu, Mie 514-8507, Japan

E-mail: katsuyas@clin.medic.mie-u.ac.jp

Key words: Cellular apoptosis susceptibility, apoptosis, hepatocellular carcinoma might play an important role in the development of human HCCs.

\section{Introduction}

The cellular apoptosis susceptibility protein (CAS) is the human homologue of the product of the yeast chromosome segregation gene, CSE1 (1). CAS is a cytoplasmic antigen associated with microtubules and the mitotic spindle (2) and maps to human chromosome 20 (20q13) (3). CAS appears to play a role in apoptosis, since down-regulation of CAS via an antisense construct has been shown to render cells which are resistant to apoptosis normally induced by tumor necrosis factor (TNF) (3). CAS function has also been linked to cell proliferation, since CAS RNA is expressed at high levels in proliferating cells but only at low levels in cells and tissue that do not proliferate (1). Immunohistochemical studies on tissue sections demonstrated that CAS is abundant in normal proliferating cells, such as those in the testis. CAS expression may also be elevated in tissue in which apoptosis normally occurs, such as the testis and breast, which contain increased amounts of CAS (2).

A number of different studies suggest that CAS is associated with the development of cancer and it is known that CAS is expressed at high levels in some cancer cell lines. In addition, the CAS gene is positioned on a chromosomal region that is often amplified in aggressive types of breast, colon, and bladder cancer (5). Moreover, CAS itself has been shown to be expressed at high levels in some cancer cell lines (5-7).

Cell proliferation and inhibition of apoptosis are thought to be important to the development of cancer (8-10). However, how regulation of the CAS gene may relate to cell proliferation or apoptosis in HCCs is not well understood. Therefore, in this study we looked at CAS expression and examined the relationship between CAS expression and cell proliferation and apoptosis in human HCCs.

\section{Materials and methods}

Immunoblot analysis. The human HCC cell lines, HepG2, Hep3B and Huh7, were homogenized in lysis buffer $(50 \mathrm{mM}$ Tris-HCl pH 8.0, 150 mM NaCl, 5 mM EDTA, 1\% NP-40, 
$1 \mathrm{mM}$ PMSF), and cell extracts were analyzed for total protein content by a protein assay (BioRad). Equal amounts of protein were separated by SDS-PAGE on $14 \%$ gels and then transferred to nitrocellulose membranes. After blocking with $5 \%$ milk, membranes were probed at $4^{\circ} \mathrm{C}$ overnight with goat anti-CAS polyclonal antibody (Santa Cruz Biotechnology, CA). The immunoblots were then probed with HRP-conjugated anti-goat IgG. Finally, after three washes, proteins were detected using the ECL system (Amersham, UK).

Tissue samples. HCCs and non-tumor tissue were obtained from 20 patients (16 males and 4 females; median age, 62.6 years) who underwent surgical procedures to remove HCCs at our institution. The tumor-bearing liver specimens of these patients were ten of type $\mathrm{C}$ liver cirrhosis, two of type $\mathrm{B}$ liver cirrhosis, three of type NBNC liver cirrhosis, one of type $\mathrm{C}$ chronic hepatitis, and four of type NBNC chronic hepatitis. The histological grade of HCC was well differentiated in five patients, moderately differentiated in 13 , and poorly differentiated in two. The tumor size of HCC was $<30 \mathrm{~mm}$ in seven patients, $\geq 30 \mathrm{~mm}$ but $<50 \mathrm{~mm}$ in seven patients, and $\geq 50$ $\mathrm{mm}$ in six patients. None of the HCCs were treated with chemotherapeutic drugs before surgical resection.

Immunohistochemistry. Tissue was collected after surgical removal, fixed immediately in $4 \%$ formalin, processed for one day at room temperature (RT), and then embedded in paraffin. Four-micron paraffin sections were prepared from the paraffin blocks and adhered to silanized slides. They were deparaffinized and hydrated by passage through xylene and a graded series of ethanol. Then, they were immersed in citrate buffer (pH 6.0) and autoclaved at $121^{\circ} \mathrm{C}$ for $10 \mathrm{~min}$. The sections were next incubated with methanol containing $0.3 \%$ $\mathrm{H}_{2} \mathrm{O}_{2}$ for 20 min and with normal rabbit and horse serum for 20 min at RT. The primary antibodies used in this study were a goat polyclonal anti-human CAS antibody (Santa Cruz Biotechnology) and a mouse monoclonal anti-human PCNA antibody (Oncogene Science, NY). The sections were incubated overnight at $4^{\circ} \mathrm{C}$ with primary antibodies at a $1: 100$ dilution in phosphate-buffered saline solution (PBS) containing $1 \%$ fetal bovine serum. The sections were next incubated for 30 min with biotinylated anti-goat and anti-mouse immunoglobulin diluted 1:200 in PBS, followed by avidin-biotinperoxidase complex (Vectastain ABC kit; Vector Laboratories, Burlingame, CA.) diluted 1:200 in PBS for $30 \mathrm{~min}$ at RT. After each incubation step, sections were carefully washed three times in PBS, for 5 min each time. The sections were developed in a substrate solution of $0.01 \%$ 3,3'-diaminobenzidine-hydrogen peroxide (DAB) and counterstained with Mayer's hematoxylin. Finally, they were rehydrated in ethanol, cleared in xylene and mounted. Negative controls were prepared using isotype-matched goat IgG and mouse IgG.

The stained sections were examined under magnification of $x 200$. The immunostaining intensity of CAS was analyzed semi-quantitatively using the following scoring system. A score of 1 was defined as weak staining intensity, a score of 2 was defined by moderate staining intensity and a score of 3 was defined by strong staining intensity. PCNA LI was calculated as the ratio of PCNA-positive hepatocyte nuclei to the total number of hepatic nuclei counted, which was 1,000 .
Hepatocytes showing brown nuclei with granular or homogeneous patterns were considered positive for PCNA.

In situ DNA nick end-labeling. Apoptotic cells were detected using the Apop Tag kit (Oncor Inc., Gaithersburg, MD) based on the method described by Gavrieli et al. After deparaffinization, sections were digested with proteinase $\mathrm{K}(20 \mu \mathrm{g} / \mathrm{ml})$ for $30 \mathrm{~min}$ at RT. They were treated with methanol containing $0.3 \% \mathrm{H}_{2} \mathrm{O}_{2}$ for $20 \mathrm{~min}$ and allowed to stand in a terminal deoxynucleotidyl transferase (TdT) buffer for $15 \mathrm{~min}$ at RT. They were then reacted with the TdT reaction mixture (TdT solution plus digoxigenin-11-dUTP and dATP) for $60 \mathrm{~min}$ at $37^{\circ} \mathrm{C}$. The sections were reacted with anti-digoxigen antibody peroxidase for $30 \mathrm{~min}$ at RT, visualized with DAB, and counterstained with Mayer's hematoxylin. Nuclei with clearly visible brown staining were regarded as positive. Necrotic areas and the edge of the tissue sections were not included in the counting to avoid possible immunohistochemical false positive stains. The stained sections were examined under magnification of $\mathrm{x} 400$. TUNEL LI was calculated as the percentage of TUNEL-positive nuclei among 2000 nuclei in several randomly selected areas.

Immunofluorescence. HepG2 cells were plated at a density of $5 \times 10^{4}$ cells per $76 \times 26 \mathrm{~mm}^{2}$ silanized slides (Dako, Kyoto, Japan) and grown for $24 \mathrm{~h}$. The cells were washed with phosphate buffered silanized slides (Daki, Kyoto) and grown for $24 \mathrm{~h}$. The cells were washed with phosphate buffered saline (PBS) fixed in $4 \%$ paraformaldehyde, and permeabilized for $10 \mathrm{~min}$ in PBS containing $0.2 \%$ Triton X-100 before incubation with $10 \%$ fetal calf serum in PBS. Cells were incubated with $3.0 \mu \mathrm{g} / \mathrm{ml}$ of anti-CAS antibody for $1 \mathrm{~h}$ at $4^{\circ} \mathrm{C}$ and then incubated with $2.0 \mu \mathrm{g} / \mathrm{ml}$ of fluorescein isothiocyanate-labeled goat anti-rabbit IgG (Chemicon, Temecula, CA) for $30 \mathrm{~min}$ at $4^{\circ} \mathrm{C}$. Fluorescence was observed with a laser scan microscope 410 (Zeiss, Gottingen, Germany).

Statistical analysis. The LIs are presented as the mean \pm standard deviation. The Chi-square test was used to compare the staining intensity of HCC and non-tumor tissue. The Mann-Whitney U-test was used for other group comparisons. $\mathrm{P}$-value $<0.05$ was regarded as statistically significant.

\section{Results}

Detection of proliferation and apoptosis in HCC tissue samples. In order to elucidate the effect of CAS expression on cell proliferation, we first looked at expression of PCNA by immunostaining to get a measure of proliferation in $\mathrm{HCC}$ tissue as compared to normal tissue. The PCNA LI in HCC $(26.24 \pm 9.56 \%)$ was significantly higher than that of nontumor tissue $(11.08 \pm 3.88 \%$; $<<0.01)$. This result indicates that there is an augmentation of proliferative activity of tumor cells compared to non-tumor tissue.

In order to elucidate the effect of CAS expression on apoptosis, apoptosis was studied by using the in situ nick endlabeling (TUNEL) method. As expected, we found that DNA frag-mentation was confined to the nuclei and that pretreatment with DNase I resulted in intense TUNEL labeling of all nuclei. As expected, control tissue sections incubated in 


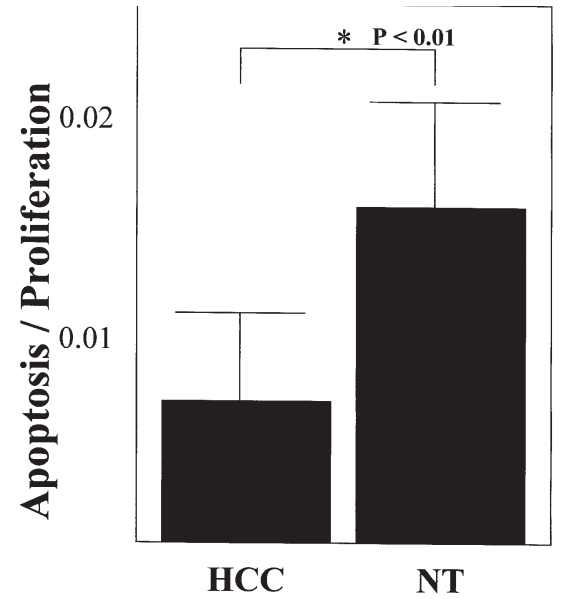

Figure 1. PCNA LI/TUNEL LI ratio in HCC and non-tumor tissue.

\section{HepG2 Hep3B Huh7 \\ CAS
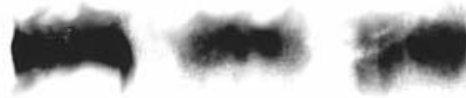 \\ $\leftarrow 116 \mathrm{kDa}$}

Figure 2. Specificity of anti-CAS in four human cell lines. The specificity of the anti-CAS antibody used in immunohistochemical analysis was confirmed in human cell lines HepG2, Hep3B, Huh7 and PLC/PRF/5. The anti-CAS antibody reacted with a single 116-kDa band, consistent with the presence of CAS protein, in all cell lines.

the absence of TdT did not show nuclear staining. The TUNEL LI for HCC cells $(1.68 \pm 0.73 \%)$ was significantly higher than that of non-tumor tissue $(0.96 \%$; $p<0.01)$. This result indicates an augmentation of apoptosis in tumor cells as compared to non-tumor tissue. However, the ratio of TUNEL LI/PCNA LI in HCC cells was significantly lower than that found in nontumor tissue (Fig. 1).

CAS expression in human HCC cell lines. In order to better understand what triggers robust proliferation of HCC cells, we looked at CAS protein expression in several HCC cell lines (Fig. 2). The anti-CAS antibody reacted with a single 116-kDa band, consistent with the expected size of CAS. In addition, the signal intensity suggested that CAS is relatively abundant in these cells. Moreover, the immunoblotting data suggest that the anti-CAS antibody will be useful for immunologic detection of CAS. In order to look at expression of CAS in cells, we next performed immunofluorescence staining of CAS protein in HepG2 cells. We found that CAS protein was predominantly detected in the cytoplasm but was also detectable in perinuclear lesions of HCC cells (Fig. 3).

CAS expression in human HCC tissue. The expression and intracellular localization of CAS protein was examined by immunohistochemistry using antibodies that specifically recognize human CAS. Our analysis showed that CAS was expressed in all HCC and non-tumor liver tissue. Similar to our results with HCC cell lines, CAS immunoreactivity in HCC tissue was predominant in the cytoplasm, appearing as a diffuse and homogeneous pattern. The level of CAS in tumor

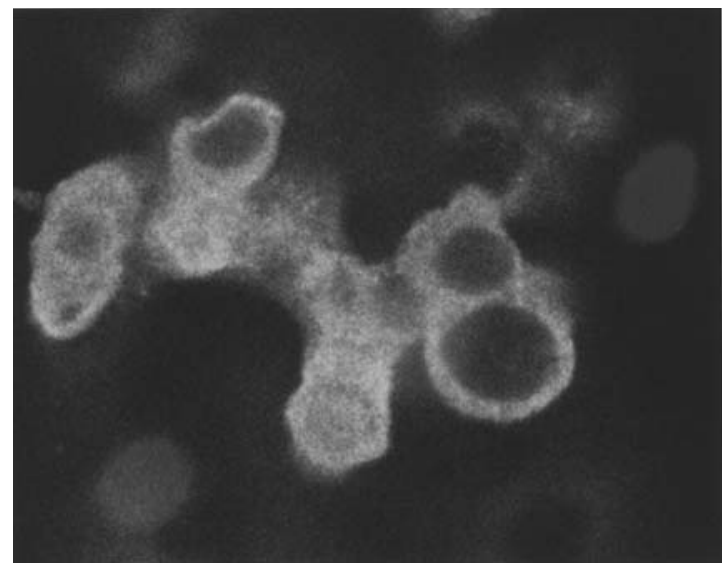

Figure 3. Immunofluorescence detection of CAS protein in HepG2 cells. CAS protein was detected predominantly in the cytoplasm but was also detected in perinuclear lesions in HCC cells.

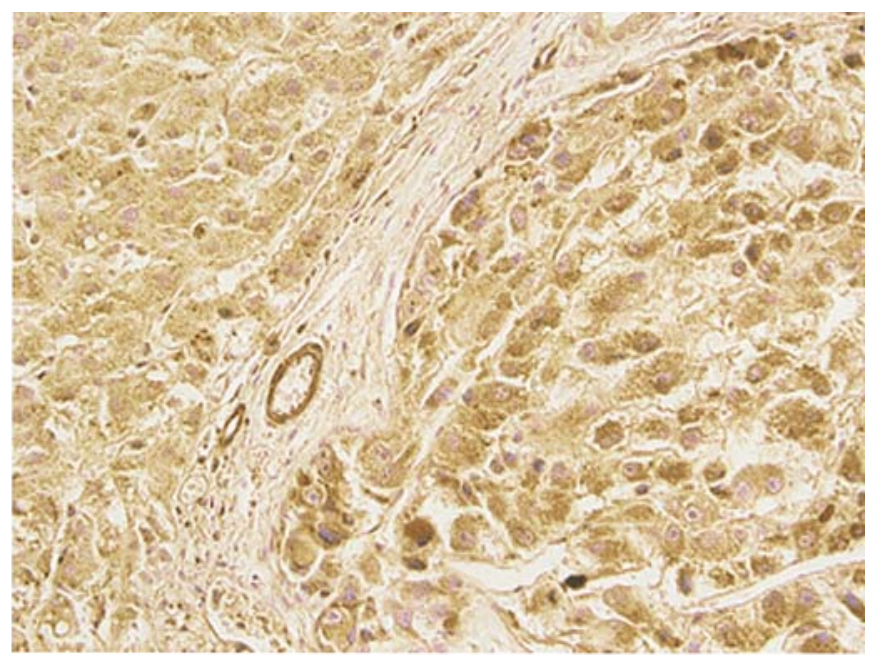

Figure 4. Immunohistochemical detection of human HCCs with anti-CAS antibody. The fibrous boundary between the HCC (left) and surrounding non-cancerous tissue (right) is apparent. Note that HCC cells are weakly stained for CAS, in contrast to non-tumor cells (original magnification $\mathrm{x} 100)$.

tissue appeared to be higher than in non-tumor tissue, since overall, we observed stronger staining in tumor tissue than in non-tumor tissue (Fig. 4).

In comparing detection of CAS in tissue from different tissue, we found that CAS staining was weak in sections from ten patients, moderate in five patients, and strong in the remaining five patients' tumor sections. In contrast, for nontumor tissue sections from the same patients, weak staining was observed for tissue from 15 patients and moderate staining was observed in tissue from five patients. The staining intensity score for CAS in HCC $(1.8 \pm 0.9)$ was significantly higher than that in non-tumor tissue $(1.3 \pm 0.4 ; \mathrm{p}<0.05)$ (Fig. 5). CAS expression did not have a statistically significant correlation with either the grade of differentiation or tumor size in the HCCs.

The average score of PCNA LI in the weak CAS staining intensity set was $21.89 \pm 7.57$, and that of moderate and strong sets was $30.58 \pm 8.86$ in HCCs. There was a significant tendency to a positive correlation between CAS and PCNA LI 


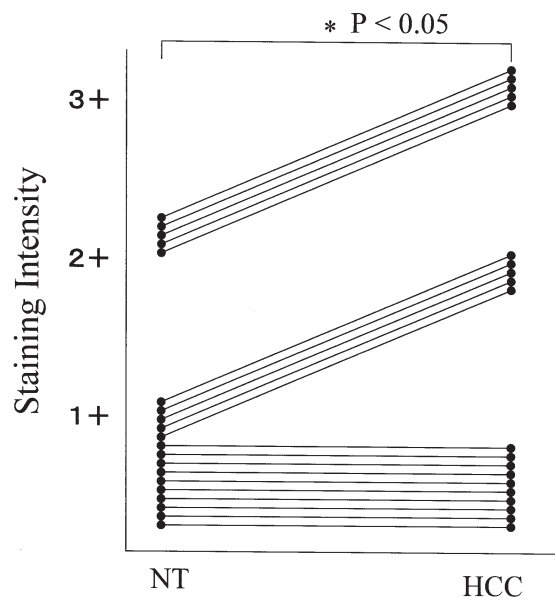

Figure 5. Comparison of CAS staining intensity in HCCs vs. non-tumor tissue. In 10 cases (50\%), CAS was detectable at higher levels in HCCs than in non-tumor tissue $(\mathrm{p}<0.05)$. NT, non-tumor tissue; $1+$, weak staining intensity; $2+$, moderate staining intensity; $3+$, strong staining intensity.
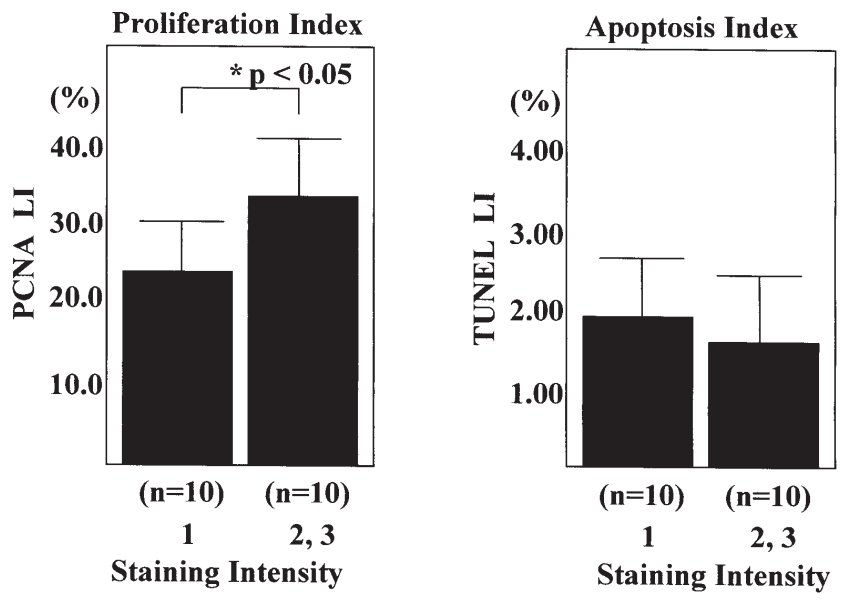

Figure 6. Measures of proliferation and apoptosis in HCC cells. PCNA LI data are expressed as the number of PCNA-positive nuclei per 1000 nuclei analyzed according to the staining intensity of CAS. The mean PCNA LI tended to increase with increasing CAS staining intensity $(\mathrm{p}<0.05)$. TUNEL LI data are expressed as the number of TUNEL-positive nuclei per 2000 nuclei analyzed according to the staining intensity of CAS. The mean TUNEL LI tended to decrease with increasing CAS staining intensity.

in HCCs $(p<0.05)$. The average score of TUNEL LI in the weak CAS staining intensity set was $1.89 \pm 0.63$, and that of moderate and strong sets was $1.47 \pm 0.72$ in HCCs. Finally, there was an inverse correlation between CAS and TUNEL LI, although the correlation did not reach statistical significance (Fig. 6).

\section{Discussion}

A better understanding of how HCCs develop could help our ability to treat the disease. In this study, we looked at the relationship between CAS expression, cell proliferation and apoptosis in HCC cell lines and tissue. As expected from the results of other studies (11-13), we found that PCNA LI, a measure of cell proliferation, was significantly higher in HCC than in non-tumor tissue. These data suggest that HCC is associated with increased cell proliferation.
It then becomes very important to examine the status of apoptotic cell death in HCC in order to understand the overall levels of cell turnover in HCC. We found that TUNEL LI was significantly higher in HCC than in non-tumor tissue, a result that is in agreement with previous studies (recent last 2 years) (8-10). However, the ratio of TUNEL LI/PCNA LI that we observed in HCC was lower than that of non-tumor tissue. Collectively, the results suggest that carcinogenesis of HCC is related to a relative increase in proliferative activity and a concurrent relative reduction of the rate of apoptotic cell death.

A previous study showed that there is a positive correlation between the PCNA index and the apoptosis index and, thus, that apoptosis may play an important role in tumor growth in less-differentiated HCCs. Furthermore, at all stages of hepatocarcinogenesis, the rate of cell proliferation was higher than the rate of apoptosis, allowing a preferential net gain of neoplastic cells, and the rate of apoptosis was significantly increased in small HCCs (8-10). This evidence suggests that a rapid turnover of cancer cells may be critically important to the development of HCCs. Thus, study of the molecular mechanism that controls the decision to undergo proliferative activity, rather than apoptosis, takes on new importance in the effort to understand HCC.

Proliferation and apoptosis are opposing cellular mechanisms; one leads to multiplication and the other to elimination of cells. Although proliferation and apoptosis can function independently of one another, the two pathways are often linked. Given the connections between cell-cycle regulation and apoptosis, it is perhaps not surprising that some proteins play roles in both mechanisms and are associated with, or may even cause cancer. A well-known example is the p53 gene, which acts in the G1 checkpoint and is also necessary for apoptosis. Many types of cancer involve loss of p53 function. Like p53, CAS, the human homologue of yeast CSE1, is thought to be associated with both apoptosis and cell proliferation, and to play a role in cancer (6).

CAS is located on human chromosome $20 q 13$ (3), a region with a remarkable degree of instability in a variety of tumors. Amplification of 20q13 occurs at the level of several independent amplicons, with CAS present in one of them (14). It has been observed that CAS and $20 \mathrm{q} 13$ amplification is also frequent in colon cancer, in which these amplifications correlate with a high degree of genetic instability (15).

CAS expression appears to be upregulated in a variety of cancer and is not limited to tumors that contain amplification of the CAS gene (7). Immunohistochemistry with anti-CAS antibodies has confirmed that the expression of CAS correlates with the development of cancer (7). In our study, CAS expression was confined to the cytoplasm and appeared as a diffuse but homogeneous staining pattern. In most proliferating tissue and cells, CAS, which has a Ran binding domain, is predominantly a cytoplasmic protein. The small ras-like protein, Ran, and some Ran-binding proteins have been shown to be involved in control of the spindle checkpoint, suggesting that CAS may have a direct function in checkpoint control $(16,17)$. The mitotic checkpoint, which monitors genomic stability during cell division, is frequently disrupted in a variety of cancer types. Collectively, previous studies and this study provide strong evidence that CAS in involved in 
hepatocarcinogenesis, an effect that may be mediated by an effect on checkpoint control.

We also questioned whether there is a correlation between CAS staining intensity of proliferation activity in human HCC tissue. To study this, we used detection of PCNA as a measure of proliferation. We found that there was a positive significant tendency toward a positive correlation between the staining intensities of CAS and PCNA LI in HCCs. This result suggests that CAS expression correlates with cell proliferation and development of HCC.

Many cancer-associated proteins require highly regulated nuclear transport, and changes in the nuclear-transport patterns of proteins such as p53 are associated with the development of cancer (8). CAS has been implicated in nuclear-to-cytoplasmic reshuffling of importin $\alpha$, which is itself necessary for nuclear transport of several proliferation activating proteins, transcription factors, oncogene and tumor suppressor gene products, including p53 and BRCA1. Over expression of CAS may interfere with the strictly regulated nuclear transport of oncogenes and/or tumor suppressors. This strongly supports the hypothesis that interference with the nuclear-transport function of CAS plays a role in many types of cancer.

It has also been shown that CAS is necessary for TNFinduced apoptosis and that depletion of CAS can render cells resistant to TNF (18). We analyzed the correlation in human HCC tissue between the expression intensity of CAS and apoptotic events, which were detected using the TUNEL method. Our results showed an inverse correlation between CAS and TUNEL LI, although the correlation did not reach statistical significance. These results strongly suggest that other proteins correlate with apoptosis resistance in human HCC.

Expression of CAS has also been examined in other malignancies. In benign and malignant cutaneous melanocytic lesions, for example, expression of CAS correlates with advanced stages of melanoma and the data suggest that antiCAS antibodies may be useful for diagnosis of melanoma and possibly as a prognostic factor in cutaneous melanocytic lesions (19). In lymphoid neoplasms, high levels of expression of CAS correlate with proliferation of normal and malignant lymphoid cells, and detection of CAS identifies a higher proportion of proliferating and malignant cells than the marker, Ki-67. Further evaluation of CAS may point to the utility of antiCAS antisera as a diagnostic marker (20). The authors of these reports point out that there is a tendency towards a positive correlation between CAS expression and proliferative activity, and also note the important biological role for CAS in a variety of malig-nancies.

In conclusion, we observed a positive correlation between CAS expression and proliferative activity, but did not observe a significant correlation between CAS expression and the grade of differentiation or tumor size. We conclude that CAS is significantly over expressed in human HCC lesions; that it may be associated with cell proliferation rather than apoptosis and, finally, that it might play an important role in the development of human HCC.

\section{References}

1. Brinkmann U, Brinkmann E, Gallo M and Pastan I: Cloning and characterization of a cellular apoptosis susceptibility gene, the human homologue to the yeast chromosome segregation gene CSE1. Proc Natl Acad Sci USA 92: 10427-10431, 1995.
2. Scherf U, Pastan I, Willingham MC and Brinkmann U: The human CAS protein, which is homologous to the CSE1 yeast chromosome segregation gene product, is associated with microtubules and mitotic spindle. Proc Natl Acad Sci USA: 2670-2674, 1996.

3. Brinkmann U, Gallo M, Polymeropoulos MH and Pastan I: The human CAS (cellular apoptosis susceptibility) gene mapping on chromosome 20q13 is amplified in BT474 breast cancer cells and part of aberrant chromosomes in breast and colon cancer cells. Genome Res 6: 187-194, 1996.

4. Brinkmann U, Brinkmann E, Bera TK, Wellmann A and Pastan I: Tissue-specific alternative splicing of the CSE1L/CAS (Cellular Apoptosis Susceptibility) gene. Genomics 58: 41-49, 1999.

5. Hino N, Higashi T, Nouso K, Nakatsukasa $H$ and Tsuji $T$ : Apoptosis and proliferation of human hepatocellular carcinoma. Liver 16: 123-129, 1996.

6. Behrens P, Brinkmann U and Wellmann A: CSE1L/CAS: its role in proliferation and apoptosis. Apoptosis 8: 39-44, 2003.

7. Ulrich Brinkmann: Human genetics 1998: Apoptosis CAS, the human homologue of the yeast chromosome-segregation gene CSE1, in proliferation, apoptosis, and cancer. Am J Hum Genet 62: 509-513, 1998.

8. Soini Y, Virkajarvi N, Lehto VP and Paakko P: Hepatocellular carcinomas with a high proliferation index and a low degree of apoptosis and necrosis are associated with a shortened survival. BJC 73: 1025-1030, 1996.

9. Park YN, Chae KJ, Kim YB, Park C and Theise N: Apoptosis and proliferation in hepatocarcinogenesis related to cirrhosis. Cancer 92: 2733-2738, 2001.

10. Ito Y, Matsuura N, Sakon M, Takeda T, Umeshita K, Nagano H, Nakamori S, Dono K, Tsujimoto M, Nakahara M, Nakao K and Monden M: Both cell proliferation and apoptosis significantly predict shortened disease-free survival in hepatocellular carcinoma. BJC 81: 747-751, 1999

11. Kitamoto M, Nakanishi T, Kira S, Kawaguchi M, Nakashio R, Suemori S, Kajiyama G, Asahara T and Doni K: The assessment of proliferating cell nuclear antigen immunohistochemical staining in small hepatocellular carcinoma and its relationship to histologic characteristics and prognosis. Cancer 15: 18591865,1993

12. Nakajima T, Kagawa K, Ueda K, Ohkawara T, Kimura H, Kakusui M, Deguchi T, Okanoue T, Kashima K and Ashihara T: Evaluation of hepatic proliferative activity in chronic liver disease and hepatocellular carcinomas by proliferating cell nuclear antigen (PCNA) immunohistochemical staining of methanol-fixed tissues. J Gastroenterol 29: 450-454, 1994.

13. Terada $T$ and Nakanuma Y: Expression of apoptosis, proliferating cell nuclear antigen, and apoptosis-related antigens (bcl-2, c-myc, Fas, Lewisy and p53) in human cholangiocarcinomas and hepatocellular carcinomas. Pathol Int. 46: 764$770,1996$.

14. Tanner MM, Tirkkonen M, Kallioniemi A, Collins C, Stokke TY, Karhu R and Kowbel D: Increased copy number at 20q13 in breast cancer: defining the critical region and exclusion of candidate genes. Cancer Res 54: 4257-4260, 1994.

15. Ried T, Knutzen R, Steinbeck R, Blegen H, Schrock E Heselmeyer K and du Manoir S: Comparative genomic hybridization reveals a specific pattern of chromosomal gains and losses during the genesis of colorectal tumors. Genes Chromosomes Cancer 15: 234-235, 1996.

16. Wiese C, Wilde A, Moore MS, Adam SA, Merdes A and Zheng Y: Role of importin-beta in coupling Ran to downstream targets in microtubule assembly. Science 291: 653-656, 2001.

17. Wilde A and Zheng Y: Stimulation of microtubule aster formation and spindle assembly by the small GTPase Ran. Science 284:1359-1362, 1999.

18. Brinkmann U, BrinkmannE, Gallo M, Scherf U and Pastan I: Role of CAS, a human homologue to the yeast chromosome segregation gene CSE1, in toxin and TNF-mediated apoptosis. Biochemistry 35: 6891-6899, 1996.

19. Boni R, Wellmann A, Man YG, Hofbauer G and Brikmann U: Expression of the proliferation and apoptotic-associated CAS protein in benign and malignant cutaneous melanocytic lesions. Am J Dermatopathol 21: 125-128, 1999.

20. Wellmann A, Krenacs L, Fest T, Scherf U, Pastan I, Raffeld M and Brinkmann U: Localization of the cell proliferation and apoptosis associated CAS protein in lymphoid neoplasms. Am J Pathol 150: 25-30, 1997. 